\title{
Pharmacotherapy of Disruptive Behavior and Item Changes on a Standardized Rating Scale: Pooled Analysis of Risperidone Effects in Children with Subaverage IQ
}

\author{
Michael Aman, Ph.D., ${ }^{1}$ Jan Buitelaar, M.D., ${ }^{2}$ Goedele De Smedt, M.D., ${ }^{3}$ \\ Robert Wapenaar, Ph.D., ${ }^{3}$ and Carin Binder, M.B.A. ${ }^{4}$
}

\begin{abstract}
Background: Disruptive behavior disorders (DBDs), excluding attention deficit/hyperactivity disorder (ADHD), are characterized by a repetitive pattern of antisocial, aggressive, and defiant behavior involving major violations of age-appropriate norms, resulting in significant functional impairment. Risperidone is licensed for the treatment of DBDs in children, adolescents, and adults in several countries. The aim of this study was to determine the effect of risperidone in a clinical setting on the symptom items of the Nisonger Child Behavior Rating Form (N-CBRF), used for the assessment of DBD patients.

Method: Data from two 6-week, randomized, double-blind, placebo-controlled trials of risperidone oral solution $(0.02-0.06 \mathrm{mg} / \mathrm{kg} / \mathrm{day})$ in children with DBDs and subaverage IQ (mild, moderate mental retardation and borderline IQ) were pooled for analysis.

Results: Risperidone produced improvement in both the Social Competence and the Problem Behavior N-CBRF subscales. Risperidone reduced symptoms in the Problem Behavior subscales (e.g., Conduct Problem, Insecure/Anxious) but also improved positive behaviors on the Social Competence subscales. Unlike most problem-behavior items, certain items reflecting "Affective insecurity" (e.g., shy, timid; clings to adults; crying, tearful episodes) failed to improve. This was also true of social disinterest and certain rituals. No items showed any worsening of symptoms with active medication.

Conclusion: Whereas most categories of problem behavior improved with risperidone, items reflecting "affective insecurity" and some infrequently endorsed items were unaffected in these children with DBDs and subaverage IQ. These data may provide a more refined knowledge of risperidone's therapeutic effects in such children.
\end{abstract}

1The Nisonger Center, Ohio State University, Columbus, Ohio.

2University Medical Centre, Nijmegen, The Netherlands.

3Johnson and Johnson, Pharmaceutical Research and Development L.L.C., Beerse, Belgium.

4 Janssen-Ortho Inc., Toronto, Ontario, Canada.

The first two authors serve on advisory boards for Janssen Pharmaceutica, and Michael Aman holds a research contract from Janssen Pharmaceutica. Goedele De Smedt is an employee of Tibotec Virco, Belgium; Robert Wapenaar is employed by Janssen-Cilag B.V., The Netherlands, and Carin Binder is employed by Janssen-Ortho Inc., Toronto, Ontario. 


\section{INTRODUCTION}

Cord ONDUCT DISORDER, oppositional defiant disorder, and disruptive behavior disordernot otherwise specified (NOS) (collectively referred to in this paper as disruptive behavior disorders (DBDs)) are among the most widespread forms of psychopathology in children and adolescents (Steiner 1997). These disorders affect approximately $6 \%$ of children and adolescents. It is widely accepted that behavior problems occur more frequently in children with mental retardation than in the general population (Einfeld and Aman 1995; Buitelaar 2002). DBDs are most common in young boys and those with low intellectual functioning (IQ under 84), with prevalence rates as high as $64 \%$ in children with severe mental retardation (Bauermeister et al. 1994; Gillberg et al. 1986). DBDs are characterized by repetitive patterns of antisocial, aggressive, and defiant behaviors that violate age-appropriate expectations or norms, resulting in significant impairment in functioning. The defining symptoms of DBDs specified in the Diagnostic and Statistical Manual of Mental Disorders, 4th edition (DSM-IV) are very similar to those of conduct disorder incorporated in International Classification of Diseases (ICD-10) (American Psychiatric Association 2000; World Health Organization 1992). Overt aggression is the most common presenting symptom for child psychiatric patients with DBDs (Connor and Steingard 1996).

Ideally, management of DBDs is holistic; treatment should involve behavioral therapy or psychotherapy and parental counseling, and it is often complimented by pharmacotherapy for symptom control (Kazdin 1997). As can be seen by the wealth of literature in this area, off-label use of medications is common, including conventional antipsychotics, psychostimulants, mood stabilizers, antidepressants, and presynaptic noradrenergic agonists.

Risperidone is a second-generation atypical antipsychotic medication, and is licensed in several countries for treating DBDs in children, adolescents, and adults. Results from two 6-week, double-blind, randomized, placebo-controlled trials and three 1-year, open-label trials have shown risperidone $(0.02-0.06 \mathrm{mg} / \mathrm{kg} /$ day $)$ to be a well-tolerated and efficacious treatment for DBDs in children with IQs ranging from borderline intellectual functioning to moderate impairment (Aman et al. 2002; Snyder et al. 2002; Turgay et al. 2002; Findling et al. 2004).

In all of these studies, the Nisonger Child Behavior Rating Form (N-CBRF) (Aman et al. 1996) was used, along with other behavior measurement tools, to determine the efficacy of risperidone in treating disruptive behavioral symptoms. The N-CBRF is an informant behavior rating scale constructed for assessing psychopathology and maladaptive behaviors in children and adolescents with developmental disabilities and/or borderline IQs (Aman et al. 1996). The N-CBRF comprises two sections: Social Competence and Problem Behavior, with subscales within each.

The aim of this study was to determine which symptoms, as represented by items on the NCBRF, responded to treatment with risperidone.

\section{METHODS}

\section{Study design}

This was a pooled analysis of N-CBRF item ratings from two 6-week, randomized, doubleblind, placebo-controlled trials in which the safety and efficacy of oral risperidone solution (0.02-0.06 mg/kg/day) were assessed in children with DBDs. Full particulars of trial design, as well as inclusion/exclusion, can be found in the cited publications (Aman et al. 2002; Snyder et al. 2002; designated here as RIS-USA-93 and RIS-CAN-19, respectively).

\section{Behavioral outcome variables}

The studies used the parent (as opposed to the teacher) version of the N-CBRF, which has the following subscales: Part A (Social Competence): 1. Compliant/Calm (6 items), 2. Adaptive/Social (4 items); Part B (Problem Behavior): 1. Conduct problem (16 items), 2 . Insecure/Anxious (15 items), 3. Hyperactive (9 items), 4. Self-Injury/Stereotypic (7 items), 5. Self-Isolated/Ritualistic (8 items), and 6. Overly Sensitive (5 items). The Social Competence items are scored on a 4-point Likert scale ranging from 0 (not true) through 3 (completely or always 
true). Each Problem Behavior item is also scored on a 4-point scale, ranging from 0 (behavior did not occur or was not a problem) through 3 (behavior occurred a lot or was a severe problem). Although the Conduct Problem subscale was the primary outcome measure for the original reports, no data from this subscale are presented here, as the emphasis of this analysis is on other behaviors affected by risperidone. Copies of the instrument are available free of charge in PDF format from the senior author (MGA) or from the Appendix. The analysis described in this paper focuses on an item analysis of the N-CBRF (Snyder et al. 2002; Aman et al. 2002).

\section{Subjects}

The studies involved children 5-12 years of age inclusive, with clinician-assessed DSM-IV diagnosis of conduct disorder, oppositional defiant disorder, or disruptive behavior disorder-not otherwise specified (NOS) who had a DSM-IV Axis II diagnosis of borderline intellectual functioning or mild-to-moderate mental retardation (IQ in the range of 36-84) and a parent-assessed rating of at least 24 in the Conduct Problem subscale of the N-CBRF. Any pervasive developmental disorder, schizophrenia or other psychotic disorders, head injury as a cause of the mental impairment, or seizure disorder requiring medication were all exclusionary criteria. Previous exposure to risperidone, history of tardive dyskinesia or neuroleptic malignant syndrome, presence of human immunodeficiency virus, and serious or progressive illness were also exclusion criteria. Subjects were required to be free of other medicines except for previously established regimens of psychostimulants (for ADHD), and chloral hydrate, antihistamines, or melatonin (for sleep). Such prior medication had to be in place at constant dosage for at least 30 days before the trial started, and dosage had to be held constant during the trial. Thirty-three percent of the placebo group and $42 \%$ of the risperidone-treated subjects received constant doses of stimulants during the trial. All patients included in the intent-to-treat studies were included in the pooled analysis of the N-CBRF items. For a summary of the char- acteristics and diagnostic classifications of the subjects, see Table 1 .

Subjects were recruited at a multitude of sites, and recruitment procedures differed across sites. In some cases, the participants were patients of the investigators, whereas in other cases the participants were recruited from a multitude of sources, such as special education settings, psychiatric and pediatric practices, parent newsletters, and radio advertising. Parents rated their children on the Child Symptom Inventory (CSI) (Gadow and Sprafkin, 1994), an informant rating scale designed to screen for all disorders common in childhood and adolescence. Physicians and licensed psychologists then made the diagnoses of conduct disorder, oppositional defiant disorder, or disruptive behavior disorder NOS and, if present, ADHD.

\section{Data analysis}

The two randomized, double-blind, placebocontrolled trials were combined for an analysis of the N-CBRF items. An analysis of covariance (ANCOVA) was performed on the change score (baseline to endpoint) to assess the differences between risperidone and placebo. The factors used were trial (RIS-CAN-19 or RIS-USA-93) and treatment, with baseline scores entered as a covariate. The Interactions between baseline scores and treatment were not assessed. Degrees of freedom throughout were 1 and 219 for the drug comparison. Although the 4-point Likert scale used for the individual items might suggest choice of a nonparametric statistic, we opted for the ANCOVA, because the ANCOVA model enabled us to control for subjects' baseline scores, study, and site differences. Computer-simulation studies have shown that the use of the ANCOVA model is appropriate and renders accurate alpha levels when applied to clinical ordinal data such as these, provided that the sample size is large (true here) (Sullivan and D'Augustino, Sr. 2003). Because of the large number of comparisons conducted, we set the alpha probability level at 0.01 . As this might permit some spurious items to emerge as "significant," items identified between 0.01 and 0.001 might best be viewed as reflecting statistical "trends." 
Table 1. Baseline Demographic and Psychiatric History Characteristics

\begin{tabular}{lccc}
\hline Baseline characteristics & CAN-19 & USA-93 & Pooled \\
\hline Number & 109 & 114 & 223 \\
Age (mean) & 8.7 & 8.3 & 8.5 \\
Sex (M/F) & $82 / 27$ & $93 / 21$ & $175 / 48$ \\
Race & $(75 \% / 25 \%)$ & $(82 \% / 18 \%)$ & $(79 \% / 21 \%)$ \\
$\quad$ Black & $8(7 \%)$ & $38(33 \%)$ & $46(21 \%)$ \\
Caucasian & $83(76 \%)$ & $65(57 \%)$ & $148(66 \%)$ \\
Hispanic & - & $6(5 \%)$ & $6(3 \%)$ \\
Asian & - & $1(1 \%)$ & $1(<1 \%)$ \\
Other & $18(17 \%)$ & $4(4 \%)$ & $22(10 \%)$ \\
Psychiatric history & & & \\
CD & $10(9 \%)$ & $20(18 \%)$ & $30(13 \%)$ \\
CD + ADHD & $31(28 \%)$ & $25(22 \%)$ & $56(25 \%)$ \\
DBD NOS & $4(4 \%)$ & $2(2 \%)$ & $6(3 \%)$ \\
DBD NOS + ADHD & $6(5 \%)$ & $6(5 \%)$ & $12(5 \%)$ \\
ODD & $12(11 \%)$ & $24(21 \%)$ & $36(16 \%)$ \\
ODD + ADHD & $47(43 \%)$ & $37(32 \%)$ & $84(37 \%)$ \\
Intellectual handicap & & & \\
$\quad$ Borderline & $53(48 \%)$ & $57(50 \%)$ & $710(49 \%)$ \\
Mild & $42(38 \%)$ & $37(32 \%)$ & $79(35 \%)$ \\
Moderate & $15(14 \%)$ & $20(18 \%)$ & $35(16 \%)$ \\
\hline
\end{tabular}

$\mathrm{CD}$, conduct disorder; $\mathrm{ADHD}$, attention-deficit/hyperactivity disorder; DBD NOS, disruptive behavior disordernot otherwise specified; ODD, oppositional defiant disorder.

\section{RESULTS}

\section{Subjects}

In total, 223 patients were included in the pooled-analysis sample. With the exception of ethnicity, patients in both trials (Aman et al. 2002; Snyder et al. 2002) were comparable in terms of baseline characteristics and psychiatric history (see Table 1).

\section{$N-C B R F$ item analysis}

Previously published studies (Aman et al. 2002; Snyder et al. 2002) used the Conduct Problem subscale as the primary outcome variable and showed that it was, statistically, significantly improved compared with placebo $(p<0.001)$. Therefore, this paper will focus on the other subscale items (exclusive of Conduct Problem), namely, the Social Competence subscales (Compliant/Calm and Adaptive/Social), and the remaining Problem Behavior subscales (Insecure/Anxious, Hyperactive, Self-Injury/ Stereotypic, Self-Isolated/Ritualistic, and Overly Sensitive).
Social Competence section. The Social Competence section subscale items that were most significantly improved $(p<0.001)$ with risperidone were: 1. "accepted redirection," 4. "initiated positive interactions," 10 . "been patient, able to delay," 2. "expressed ideas clearly," 5. "participated in group activities," and 7. "shared with or helped others." At the $p<0.01$ level, the significantly improved items were: 3 . "followed rules," and 8. "stayed on-task" (see Table 2). Effect sizes (presented in Table 2) for significant items ranged from 0.29 to 0.48 .

Problem Behavior section. For the Insecure/ Anxious subscale, the majority of items were markedly improved with risperidone at endpoint, compared to placebo (see Table 3). The items most significantly improved $(p<0.001)$ with risperidone were: 31 . "nervous or tense," 41. "says no one likes him or her," 42. "secretive, keeps things to self," and 55. "talks too much or too loud." Items 16. "exaggerates abilities or achievements," 21. "feels others are against him or her," 30. "lying or cheating," 48. "steals," 52. "sulks, is silent and moody," and 


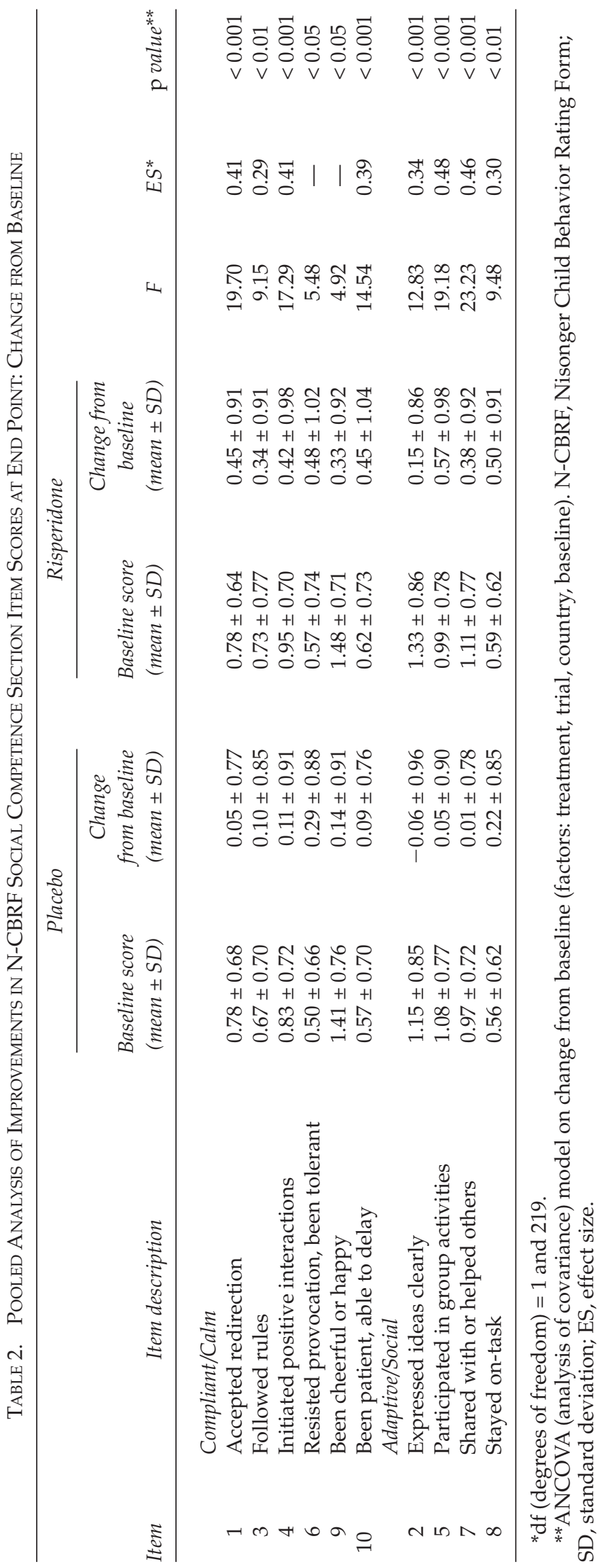




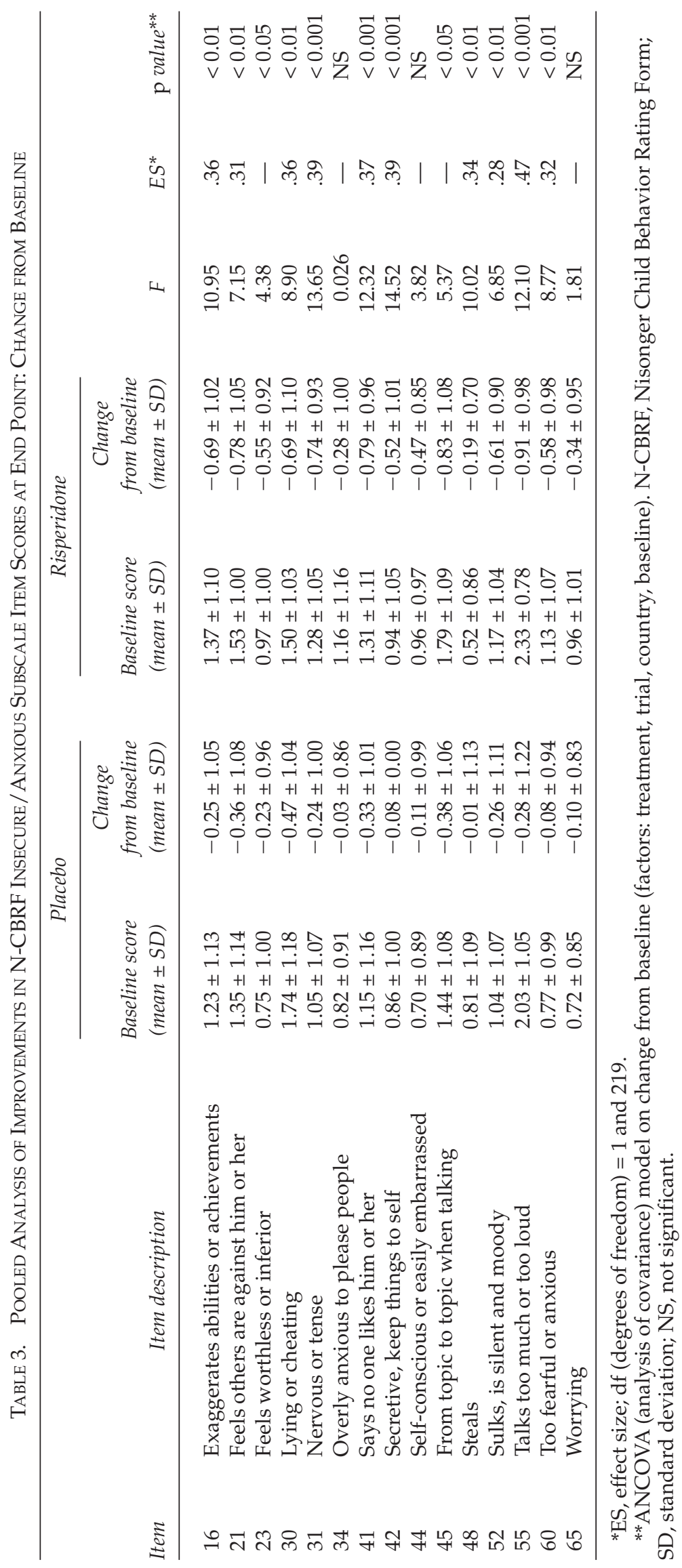


60. "too fearful or anxious" were significant at the $p<0.01$ level. Effect sizes ranged from 0.28 ("sulks") to 0.47 ("talks too much") (see Table $3)$. The items for which there was no significant difference between risperidone and placebo at endpoint were: 34 . "overly anxious to please people," 44. "self-conscious or easily embarrassed," and 65. "worrying."

On the Hyperactivity subscale, items that were significantly improved with risperidone were: 33. "overactive, doesn't sit still" [F(1219) = 12.07; Effect size (ES), 0.44), 39. "restless, high energy level" ( $\mathrm{F}=11.33$; ES, 0.42) $(p<0.001)$, and 13. "easily distracted" ( $F=9.64 ; E S, 0.38)$, 19. "fails to finish things he or she starts" ( $\mathrm{F}=$ 9.83; ES, 0.38), and 46. "short attention span" $(\mathrm{F}=9.02 ; \mathrm{ES}, 0.39)(p<0.01)$. On the Self-Injury / Stereotypic subscale, only 53. "physically harms / hurts self on purpose" (F = 10.90; ES, 0.21) was significantly improved $(p<0.01)$ with risperidone. For the Self-Isolated/Ritualistic subscale, 3 of the 8 items were significantly improved with risperidone compared to placebo at endpoint (see Table 4). These were: items 29. "isolates self from others," 37. "refuses to talk," and 49. "odd repetitive behaviors." Effect sizes ranged from 0.22 (item 49) to 0.29 (item 37). The 3 items for which there was no significant difference at endpoint between risperidone and placebo were: 1 . "disinterested or unmotivated," 18. "rituals: head rolling or floor pacing," and 47. "shy or timid behavior." On the Overly Sensitive subscale, the only significantly improved item was: 14 . "easily frustrated" ( $\mathrm{F}=$ 17.66 ; ES, 0.52) $(p<0.001)$. Other items, not assigned to a subscale, that were significantly improved with risperidone were: 51 . "sudden changes in mood" ( $\mathrm{F}=10.39 ; \mathrm{ES}, 0.43)(p<0.01)$ and 27. "irritable" $(\mathrm{F}=13.62 ; \mathrm{ES}, 0.45)(p<0.001)$.

\section{DISCUSSION}

\section{Social Competence section items}

One finding from this analysis was the impact that risperidone had on individual positive social behaviors, as shown by improvements in items on the Compliant/Calm and Adaptive/Social subscales. Based on results in Table 2 , the most dramatic effects on the Compli-
ance/Calm subscale were observed with symptoms of rule-governed behavior and parental guidance. Parents rated their children as better able to accept guidance, show patience, and follow rules. The affective items showed less effect. On the Adaptive/Social subscale, there was a significant increase in interaction, socialization, sharing, and group activities with risperidone. The improvement in social behaviors, and especially rule-governed behaviors, may lead to better integration in the home, school, and society. Children who are receptive to parental and teacher guidance may be better able to study, find suitable employment, and avoid criminal activity.

\section{Insecure/Anxious subscale items}

It is interesting that the items relating to mood symptoms, such as social anxiety ("overly anxious to please people," "self-conscious or easily embarrassed," "worrying" — see Table 3), were not very sensitive to medication. As in the case of greater socialization with medication, this deserves objective study in its own right. When more is known about the various atypical antipsychotic medications, these kinds of subtle differences in effect may be a basis for choosing one medicine over another. The lack of effect on shyness may also indicate informant discrimination between certain items and the lack of "halo effect," at least for certain items. A "halo effect," commonly quoted in the literature, occurs when a rater perceives improvements in all symptoms with medication when, actually, only a prominent symptom (often, aggression in the DBDs) has been reduced. As indicated further below, lack of change could be seen as "therapeutic" in such children.

\section{Hyperactive subscale items}

In the two trials analyzed here, up to $60 \%$ of the children with DBD were diagnosed with ADHD. The earliest antipsychotics used to treat ADHD had an effect on physical activity but not on attention. With risperidone, such a distinction was not evident, as improvements in both physical activity symptoms and attention span were rated as occurring. However, we cannot assume that perceived improvements 


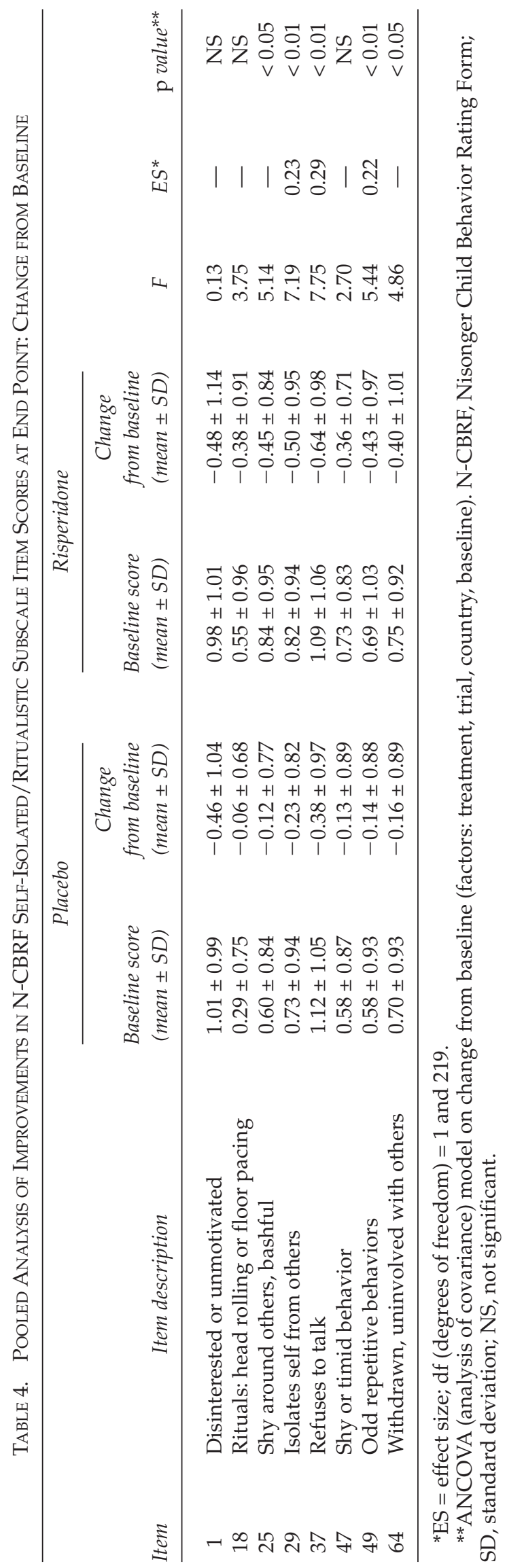


in attention span are real and, therefore, it would be helpful to gather independent data on this (e.g., from vigilance tasks) in future trials.

Thirty-three percent of placebo subjects and $42 \%$ of risperidone subjects were taking psychostimulants. However, this should not have affected our findings, as stimulant therapy had to be stabilized at least 30 days prior to the study and held constant throughout.

\section{Self-Injury/Stereotypic subscale items}

The characteristic item for stereotypic behavior, rocking, was unaffected by medication. Most self-injury items were moderately affected, while the only item to reach the 0.01 level of significance was "self-harm." However, one of the authors (MA) noted that such self-harm often appeared to be incidental and tantrum associated (i.e., a side effect of throwing oneself around in rebellion), as compared with volitional repetitive and mechanical self-harm sometimes seen in such patients.

\section{Self-Isolated/Ritualistic subscale}

On the Self-Isolated/Ritualistic subscale (see Table 4), an improvement in the "refuses to talk" and "isolates self from others" items suggests an increased interest or willingness to interact with others or engage in prosocial behaviors toward others (consistent with Social Competence section items). Response of these symptoms may also relate to the domain of negative symptoms. As in the case of prosocial behaviors mentioned above, these changes may be linked to observations in adult schizophrenic patients on risperidone medication, demonstrating a beneficial effect on negative symptoms of schizophrenia. Negative symptoms may be phenomenologically related to social withdrawal in behavioral and developmental disorders.

As noted earlier, the "shy or timid behavior," "disinterested or unmotivated," and "ritualistic: Head rolling or floor pacing" items were not affected by medication. The lack of change with "shy or timid behavior" is consistent with the affective items identified under the Insecure/Anxious subscale and adds credibility to the notion that "affective insecurity" was not modified in these children. In an odd kind of way, we might argue that stability on such items may be fruitful for children with DBDs. Some degree of anxiousness to please others may be related to a child's decision to adhere to basic rules set out by our society.

The lack of change on the "disinterested or unmotivated" and "rituals: Head rolling or floor pacing" items are not easily put in any context. Disinterest would seem to be related to the prosocial items that did show change, and the lack of effect on this item is difficult to explain. Most of the participants in this analysis had either borderline IQs or mild mental retardation. Therefore, the lack of effect on "ritualistic/ head rolling" may be a floor effect, as stereotypic behavior is often strongly related to severity of functional handicap. In fact, the modal score on this item was 0 .

\section{Other items}

There was no or limited observed effect on the Overly Sensitive items except the "easily frustrated" item. One could link the "easily frustrated" item as part of the "Conduct" constellation subscale. Most of the remaining items on this subscale ("clings to adults," "crying, tearful episodes," "overly sensitive," and "feelings easily hurt") also appear to reflect our construct of "affective insecurity." It is very interesting that such items repeatedly emerged as unaffected by the active drug.

\section{CONCLUSIONS}

Beyond any effect on conduct problem symptoms, risperidone also appeared to improve aspects of social interaction, feelings of self-worth, and reintegration. The significant improvements noted in factors measuring rule-governed behavior, isolation, socialization, and group-sharing activities may offer hope for additional changes in these aspects of human interaction.

There was a lack of effect on several moodrelated items reflecting "affective insecurity." At this stage, it is not clear if this is an area uninfluenced by risperidone or if it reflects peculiarities of this analysis. If a more general effect, it may tell us more about the action of this medication in children with DBDs. In the same 
way that a degree of anxiety in a job interview may be regarded as "healthy" (i.e., the person cares enough about the position to become nervous), some level of "insecurity" may be therapeutic in these children.

\section{ACKNOWLEDGMENTS}

This research was supported by funding from Johnson and Johnson, Pharmaceutical Research and Development, Beerse, Belgium, and the data analyses were supported by funding from Janssen-Ortho Inc., Toronto, Canada.

\section{REFERENCES}

Aman MG, De Smedt G, Derivan A, Lyons B, Findling RL, and the Risperidone Disruptive Behavior Study Group: Double-blind, placebo-controlled study of risperidone for the treatment of disruptive behaviors in children with subaverage intelligence. Am J Psychiatry 159:1337-1346, 2002.

Aman MG, Tassé MJ, Rojahn J, Hammer D: The Nisonger CBRF: A child behavior rating form for children with developmental disabilities. Res Dev Disabil 17:41-57, 1996.

American Psychiatric Association: Diagnostic and Statistical Manual of Mental Disorders, 4th edition, text revision (DSM-IV-TR). Washington (DC), American Psychiatric Association, 2000.

Bauermeister JJ, Canino G, Bird H: Epidemiology of disruptive behavior disorders. Child Adolesc Psychiatr Clin North Am 3:177-194, 1994.

Buitelaar JK: Epidemiological aspects: What have we learned over the last decade? In: Hyperactivity and Attention Disorders of Childhood, 2nd edition. Edited by Sandberg S. Cambridge: Cambridge University Press, pp. 30-63.

Connor DF, Steingard RJ: A clinical approach to the pharmacology of aggression in children and adolescents. Ann N Y Acad Sci 794:290-307, 1996.

Einfeld S, Aman M: Issues in the taxonomy of psychopathology in children and adolescents with mental retardation. J Autism Dev Disord 25:143167, 1995.
Findling RL, Aman MG, Eerdekens M, Derivan A, Lyons B, and the Risperidone Disruptive Behavior Study Group: Long-term, open-label study of risperidone in children with severe disruptive behaviors and below-average IQ. Am J Psychiatry 161:677-684, 2004

Gadow KD, Sprafkin J: Manual for the Stony Brook Child Symptom Inventory: Parent Checklist. Child Symptom Inventory: Teacher Checklist. Stony Brook (New York), Checkmate Plus, Ltd., 1994.

Gillberg C, Persson E, Grufman M, Themner M: Psychiatric disorders in mildly and severely mentally retarded urban children and adolescents: Epidemiological aspects. Br J Psychiatry 149:6874, 1986.

Kazdin AE: Practitioner review: Psychosocial treatments for conduct disorder in children. J Child Psychol Psychiatry 38:161-178, 1997.

Snyder R, Turgay A, Aman MG, Binder C, Fisman S, Carroll A, The Risperidone Conduct Study Group: Effects of risperidone on conduct and disruptive behavior disorders in children with subaverage IQs. J Am Acad Child Adolesc Psychiatry 41:1026-1036, 2002.

Steiner H: Practice parameters for the assessment and treatment of children and adolescents with conduct disorder. J Am Acad Child Adolesc Psychiatry 36 (Suppl 10):122S-139S, 1997.

Sullivan LM, D'Augustino RB, Sr: Robustness and power of analysis of covariance applied to ordinal scaled data as arising in randomized controlled trials. Statist Med 22:1317-1334, 2003.

Turgay A, Binder C, Snyder R, Fisman S: Long-term safety and efficacy of risperidone for the treatment of disruptive behavior disorders in children with subaverage IQs. Pediatrics 110:e34, 2002.

World Health Organization: ICD-10. Classification of Mental and Behavioral Disorders. Clinical Description and Diagnostic Guidelines. Geneva, World Health Organization, 1992.

Address reprint requests to: Michael Aman, Ph.D.

The Nisonger Center

Ohio State University

1581 Dodd Drive

Columbus, $\mathrm{OH}$ 43210-1257

E-mail: aman.1@osu.edu 


\section{APPENDIX A}

\section{THE NISONGER CHILD BEHAVIOR RATINGFORM}

\section{PARENT VERSION}

Child's Name:

Child's Date of Birth:

$\frac{1}{\text { month day year }}$

Rater's Name:

Date of Rating:

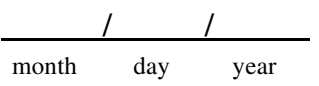

Relation of Rater to Child:

$\square$ parent [1]

$\square$ other [9]:

(please specify)

I. Please describe any special circumstances or mediating factors that may have affected the child's behavior in the recent past (the last month or two) or prevented you from making complete ratings.

II. POSITIVE SOCIAL. Please describe the child's behavior as it was at home over the last month.

IN THE LAST MONTH, THIS CHILD HAS:

1. Accepted redirection

2. Expressed ideas clearly

3. Followed rules

4. Initiated positive interactions

5. Participated in group activities

6. Resisted provocation, was tolerant

7. Shared with or helped others

8. Stayed on task

9. Was cheerful or happy

10. Was patient, able to delay

$\begin{array}{cccc}\text { Not } & \text { Somewhat or } & \text { Very or } & \text { Completely or } \\ \text { True } & \text { Sometimes } & \text { Often True } & \text { Always True } \\ {[0]} & \text { True } & {[2]} & {[3]}\end{array}$

[1]

$\begin{array}{llll}\square & \square & \square & \square \\ \square & \square & \square & \square \\ \square & \square & \square & \square \\ \square & \square & \square & \square \\ \square & \square & \square \\ \square & \square & \square \\ \square & \square & \square & \square \\ \square & \square & \square & \square \\ \square & \square & \square & \square \\ \square & \square & \square & \square\end{array}$


III. PROBLEM BEHAVIOR. For each item that describes the child's behavior as it was over the last month, circle the:
$0 \ldots$. if the behavior did not occur or was not a problem
$1 \ldots$. if the behavior occurred occasionally or was a mild problem
$2 \ldots$. if the behavior occurred quite often or was a moderate problem
$3 \ldots$ if the behavior occurred a lot or was a severe problem

For each problem that occurred, circle only the score that best describes the behavior.

\section{PLEASE DO NOT SKIP ANY QUESTIONS. IF YOU DO NOT KNOW THE ANSWER OR HAVE NOT HAD A CHANCE TO OBSERVE THE CHILD FOR A GIVEN TIME, CIRCLE THE ZERO.}

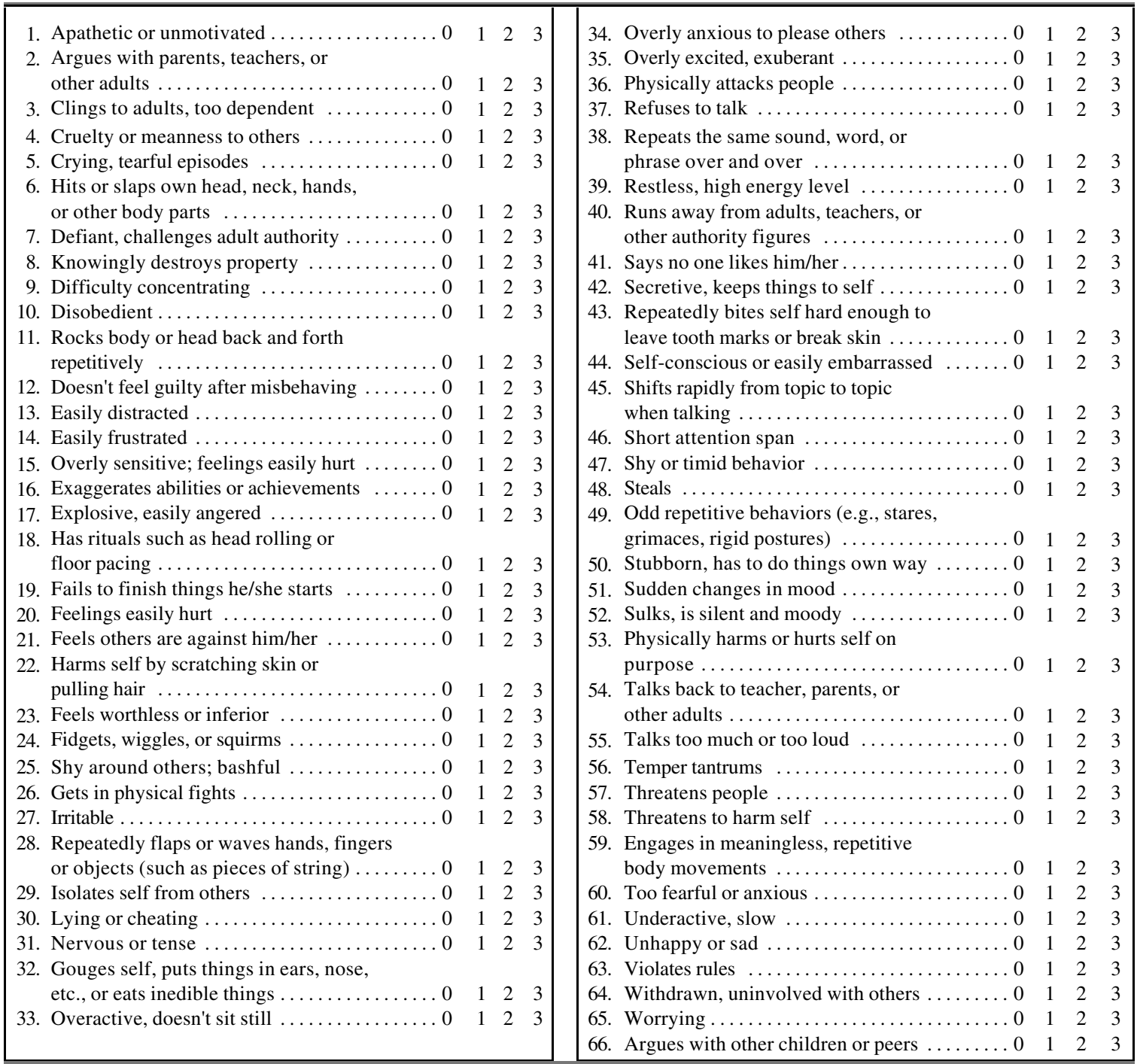

Readers are encouraged to duplicate and use this scale in their clinical practice. 


\section{THE Nisonger CHILD BEHAVIORRATINGFoRM}

\section{PARENT VERSION: SCORE SHEET}

Child's Name:

Rater's Name:

Relation of Rater to Child:
Child's Date of Birth:

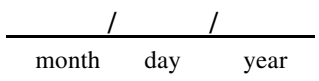

Date of Rating:

$\frac{1}{\text { month day } \quad \text { year }}$

(please specify)

INSTRUCTIONS. Transcribe the ratings from the Nisonger CBRF and write them into the "rating" column next to the appropriate item number "\#". When all ratings have been transcribed, total the columns to obtain the subscale scores.

II. POSITIVE SOCIAL

III. PROBLEM BeHAVIOR

\begin{tabular}{|c|c|c|c|}
\hline \multicolumn{2}{|c|}{$\begin{array}{l}\text { Compliant / } \\
\text { Calm }\end{array}$} & \multicolumn{2}{|c|}{$\begin{array}{l}\text { Adaptive } \\
\text { Social }\end{array}$} \\
\hline$\#$ & rating & $\#$ & rating \\
\hline 1 & & 2 & \\
\hline 3 & & 5 & \\
\hline 4 & & 7 & \\
\hline 6 & & 8 & \\
\hline 9 & & \multirow[t]{2}{*}{ Tota } & \\
\hline 10 & & & \\
\hline
\end{tabular}

Total

Conduct

\begin{tabular}{|c|c|c|c|c|c|c|c|c|c|c|c|}
\hline \multicolumn{2}{|c|}{$\begin{array}{l}\text { Conduct } \\
\text { Problem }\end{array}$} & \multicolumn{2}{|c|}{$\begin{array}{c}\text { Insecure / } \\
\text { Anxious }\end{array}$} & \multicolumn{2}{|c|}{ Hyperactive } & \multicolumn{2}{|c|}{$\begin{array}{l}\text { Self-Injury / } \\
\text { Stereotypic }\end{array}$} & \multicolumn{2}{|c|}{$\begin{array}{l}\text { Self-Isolated / } \\
\text { Ritualistic }\end{array}$} & \multicolumn{2}{|c|}{$\begin{array}{c}\text { Overly } \\
\text { Sensitive }\end{array}$} \\
\hline \# & rating & \# & rating & \# & rating & \# & rating & $\#$ & rating & \# & rating \\
\hline 2 & & 16 & & 9 & & 6 & & 1 & & 3 & \\
\hline 4 & & 21 & & 13 & & 11 & & 18 & & 5 & \\
\hline 7 & & 23 & & 19 & & 22 & & 25 & & 14 & \\
\hline 8 & & 30 & & 24 & & 32 & & 29 & & 15 & \\
\hline 10 & & 31 & & 33 & & 43 & & 37 & & 20 & \\
\hline 12 & & 34 & & 35 & & 53 & & 47 & & \multirow{3}{*}{\multicolumn{2}{|c|}{ Total }} \\
\hline 17 & & 41 & & 38 & & 58 & & 49 & & & \\
\hline 26 & & 42 & & 39 & & Total & & 64 & & & \\
\hline
\end{tabular}

\begin{tabular}{|c|c|c|l|}
\hline 26 & & 42 & \\
\hline 36 & & 44 & \\
\hline 40 & & 45 & \\
\hline 50 & & 48 & \\
\hline 54 & & 52 & \\
\hline 56 & & 55 & \\
\hline 57 & & 60 & \\
\hline 63 & & 65 & \\
\hline 66 & & \multicolumn{3}{|c|}{ Total } \\
\hline
\end{tabular}

Total

Developed by M. G. Aman, M. J. Tassè, J. Rojahn, and D. Hammer: The Nisonger CBRF: A child behavior rating form for children with developmental disabilities. Res Dev Disabil 17:41-57, 1996. 